\title{
Evidence for a Cross-Talk Between Cytosolic 5'-Nucleotidases and AMP-Activated Protein Kinase
}

\author{
Marcella Camici ${ }^{1+}$, Mercedes Garcia-Gil ${ }^{2+}$, Simone Allegrini ${ }^{1}$, Rossana Pesi ${ }^{1}$ and \\ Maria Grazia Tozzi ${ }^{1 *}$ \\ ${ }^{1}$ Unità di Biochimica, Dipartimento di Biologia, Università di Pisa, Pisa, Italy, ${ }^{2}$ Unità di Fisiologia Generale, Dipartimento di Biologia, \\ Università di Pisa, Pisa, Italy
}

Keywords: cytosolic 5'-nucleotidases I and II, AMP-activated kinase, purine cycle, body weight, muscle contraction

\section{INTRODUCTION}

The maintenance of the correct balance of nucleotide pools is essential for many vital functions (Bester et al., 2011; Garcia-Gil et al., 2018; Camici et al., 2019). The control of several enzyme activities required for nucleotide metabolism contributes to this homeostasis. Among the involved enzymes, cytosolic $5^{\prime}$-nucleotidases (NT5Cs) play a central role in the regulation of the purine nucleotide pool (Figure 1). The major NT5Cs acting on purine nucleotides are cytosolic $5^{\prime}$ nucleotidase I (NT5C1), which exerts its action mainly in skeletal muscle, and cytosolic $5^{\prime}$ nucleotidase II (NT5C2), which is ubiquitously expressed. The preferred substrate for NT5C1 is AMP, with a $K_{M}$ in the millimolar range (Hunsucker et al., 2001; Tkacz-Stachowska et al., 2005). Although preferring IMP and GMP as substrates ( $\mathrm{K}_{\mathrm{M}}$ in the micromolar range) (Tozzi et al., 2013), NT5C2 catalyses also the hydrolysis of the phosphoester bond of AMP (with a $\mathrm{K}_{\mathrm{M}}$ in the millimolar range) (Tozzi et al., 2013). The rate of the IMP-GMP cycle (Figure 1) which regulates the intracellular purine nucleotide concentrations, depends on NT5C2 activity (Barsotti et al., 2003). In fact, in the presence of high energy charge, NT5C2 catalyses the catabolism of excess IMP, synthesized by de novo or salvage pathways, while allowing for IMP and AMP accumulation in case of low energy charge (Pesi et al., 1994; Allegrini et al., 2004; Wallden and Nordlund, 2011; Camici et al., 2018). For the regulation of the AMP cycle, both NT5C1 and NT5C2 activities are involved (Figure 1). In the last decades growing evidence indicates the central "energy sensing" role played by the AMP-activated protein kinase (AMPK) (Hardie et al., 2012; Garcia and Shaw, 2017). AMPK is a heterotrimer composed of the catalytic $\alpha$ ( $\alpha 1$ or $\alpha 2)$, the regulatory $\beta$ ( $\beta 1$ or $\beta 2$ ) and the $\gamma$ subunits $(\gamma 1, \gamma 2$ or $\gamma 3)$. Alterations in the AMP:ATP ratio are perceived by the $\gamma$ subunit of AMPK which contains three AMP binding sites, two of which exchangeable with ATP (Xiao et al., 2007). The binding of AMP further increases the kinase activity of AMPK both allosterically and inhibiting its dephosphorylation (Sanders et al., 2007). The major upstream kinases that activate AMPK by phosphorylation of Thr172 (Hawley et al., 1996), are the tumour suppressor kinase LKB1 (Woods et al., 2003) and the $\mathrm{Ca}^{2+} /$ calmodulin-dependent kinase kinase $\beta$ (Hawley et al., 2005). AMPK is activated when the cellular energy charge is low and, acting on several protein targets, this protein kinase switches off the anabolic pathways that require ATP and switches on the catabolic pathways that produce ATP (Figure 1). AMPK activation brings about an increase in muscular glucose uptake and fatty acid oxidation, making AMPK activators useful tools for the treatment of type 2 diabetes (Coughlan et al., 2014). In addition, AMPK activation may be responsible for some of the tumour suppression functions of LKB1 (Hardie and Alessi, 2013). Since NT5Cs are the major responsible for the regulation of the AMP level (Kulkarni et al., 2011), it is conceivable that alterations in their activities may affect the numerous signaling pathways triggered by AMPK activation, and thus the regulation of biological doi: 10.3389/fphar.2020.609849 


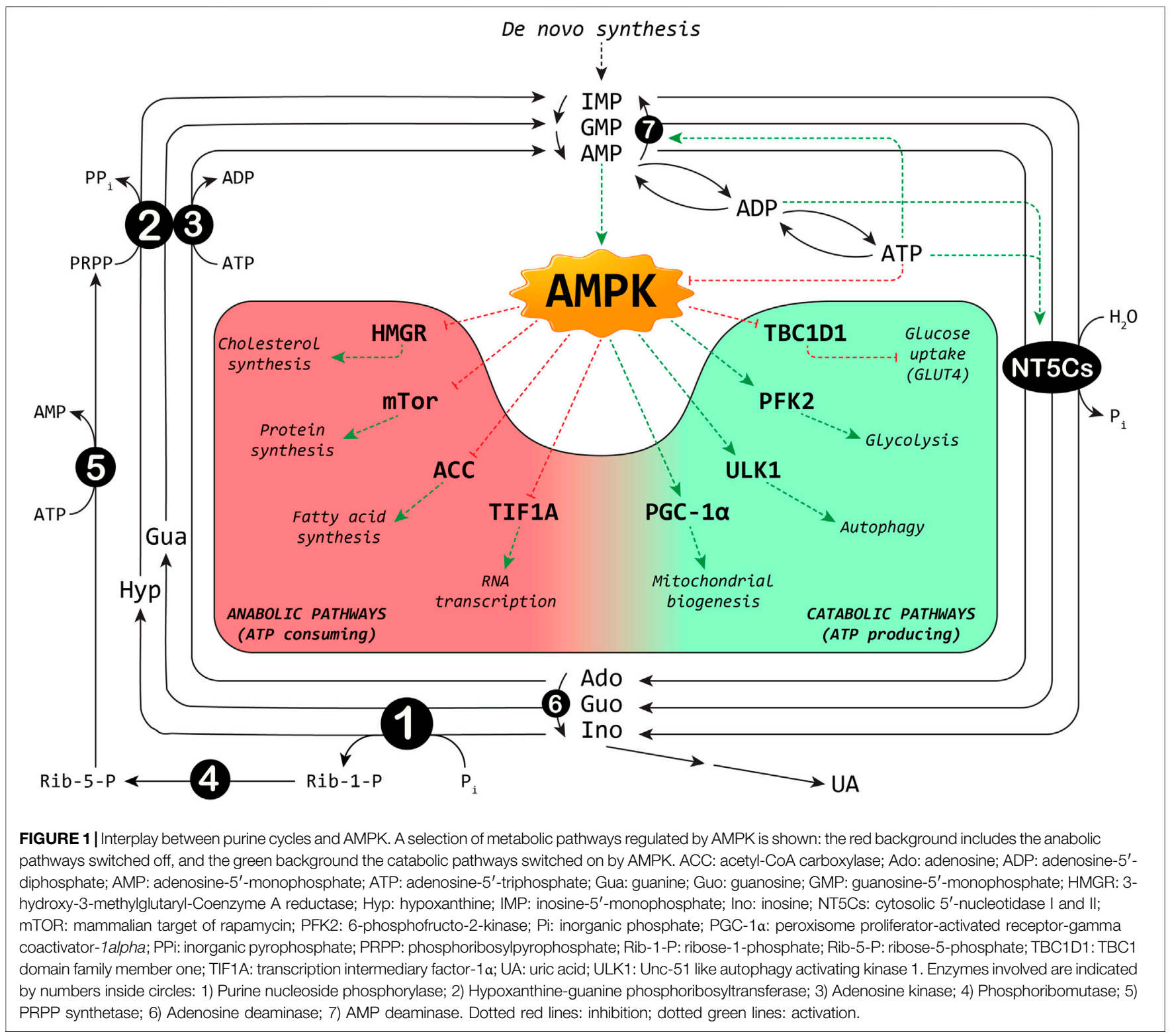

processes including muscle contraction, functioning of the nervous system, and control of body weight.

\section{In Muscle}

Gene silencing of NT5C1A by shRNA injection and electroporation in mouse tibialis anterior muscle decreased NT5C1A protein expression, increased phosphorylation of AMPK and of its substrate acetyl-CoA carboxylase (ACC), as well as glucose uptake (Kulkarni et al., 2011). Similar results were obtained by using NT5C2 siRNA in cultured human myotubes. The downregulation of NT5C2 led to an increase in the AMP: ATP ratio, an increase in AMPK phosphorylation (Thr172), and an increase in ACC phosphorylation (Kulkarni et al., 2011). Overexpression of NT5C1A in human embryonic kidney
(HEK293T) cells caused a reduction in the oligomycininduced increase in AMP and ADP concentrations and a decrease in AMPK activation (Plaideau et al., 2012). Surprisingly, NT5C1A and NT5C2 deletion were not able to potentiate AMPK activation following electrical stimulation in soleus and extensor digitorum longus (EDL) mouse muscles (Kviklyte et al., 2017). AMP:ATP or ADP:ATP ratios in the knockout resting muscles were similar to those of wild type (WT) mice, and contraction did not induce a potentiation of these ratios in the muscle of the knockout animals (Kviklyte et al., 2017). In fact, electrical stimulation induced a 4-fold increase of AMPK activity compared to the resting state both in WT and nucleotidase-deleted muscles. Also, downstream ACC phosphorylation and glucose uptake appeared to increase to 
the same extent in EDL from electrically stimulated WT and NT5C2-or NTC1A-knockout mice (Kviklyte et al., 2017). In addition, the effects of the combination of NT5C1 deletion plus inhibition of AMP deaminase on AMP:ATP ratio and AMPK activity, measured in resting and electrically-stimulated EDL muscle were not different between muscle from WT and knock-out animals (Kviklyte et al., 2017). The authors hypothesized that, during contraction, fluxes through nucleotidases might be too reduced to influence AMP levels and concluded that pharmacological inhibition of AMPmetabolizing enzymes might not be useful for promoting AMPK activation and glucose uptake in muscle of type-2 diabetic patients.

\section{In Nervous System}

In human neural progenitor cells (hNPCs), NT5C2 knockdown by siRNA increased AMPK protein expression and phosphorylation, and surprisingly, phosphorylation of $40 \mathrm{~S}$ ribosomal protein S6 (RPS6), without modification in RPS6 expression. It also altered transcription of several genes involved in protein translation (Duarte et al., 2019). RPS6 correlates with mammalian target of rapamycin complex 1 (mTORC1) activation and it is frequently used to estimate the rate of protein translation (Biever et al., 2015). HEK293T cells overexpressing NT5C2 were used to further investigate the association between NT5C2 and the regulation of AMPK and RPS6. Duarte et al. (2019) found a decrease in phosphorylated AMPK but not in total AMPK in these cells, and a decrease in total RPS6 protein associated with $300 \%$ increase in RPS6 phosphorylation. Therefore, the effect of NT5C2 on RPS6 in HEK293T cells was opposite to that observed in hNPCs. The authors suggested that the increase in RPS6 phosphorylation observed in hNPCs as a consequence of NT5C2 knockdown could be ascribed to a negative feedback loop leading to increased protein synthesis after an initial arrest in protein synthesis, already described during recovery in muscle (Dreyer et al., 2006). Indeed, endurance exercise in humans increased AMPKa2 activity and immediately decreased protein synthesis. This was followed by increased p70S6K phosphorylation, and increased protein synthesis during the recovery period, $2 \mathrm{~h}$ after a bout of exercise (Dreyer et al., 2006). It is worthy to note that since protein synthesis has not been directly measured in hNPCs, it is impossible to know whether the increase in phospho-RPS6 observed after NT5C2 knockdown (Duarte et al., 2019) does reflect a raise in protein synthesis. Conversely, protein synthesis was dramatically lower in NT5C2- knockdown human lung carcinoma (A549) cells compared to control cells (Pesi et al., 2018), but no modification of AMPK activity has been found in these cells, probably as a consequence of an inactivating mutation of LKB1 in A459 cells (Zhong et al., 2006).

NT5C2 is associated with disorders characterized by psychiatric and psychomotor disturbances such as hereditary spastic paraplegias (HSP) (Garcia-Gil et al., 2018), schizophrenia (Cross-Disorder Group of the Psychiatric Genomics, 2013; Duarte et al., 2016; Duarte et al., 2019) and Parkinson disease. The aberrantly spliced NT5C2 described by Elsaid et al. (2017) in individuals affected by HSP showed substantial reduction in expression level in the in vitro study, indicating marked instability of the mutant NT5C2 protein. The authors suggest that homozygous alteration in NT5C2 might be necessary to produce central white matter developmental defects (Elsaid et al., 2017). It is interesting to note that knockdown of the NT5C2 homologue in Drosophila melanogaster was associated with abnormal climbing behavior when driven by a neuronal promoter, supporting a role for NT5C2 in motility (Duarte et al., 2019). The mechanisms underlying the pathological effects of NT5C2 mutations are unknown. It could be interesting to obtain information not only on the levels of expression and/or activity of NT5C2, but also on the possible variations of AMP:ATP ratio which could lead to an upregulation of AMPK. A permanent activation of AMPK could result in abnormal development of the nervous system. Indeed, AMPK activation induces apoptosis in hippocampal and neuroblastoma cells (Pesi et al., 2000; Garcia-Gil et al., 2003), and reduces axonal growth (Williams et al., 2011). Moreover, AMPK hyper-activation in differentiated primary neurons reduces the number of synapses and leads to a loss of neuronal network functionality (Domise et al., 2019) and AMPK signaling has been associated with amyotrophic lateral sclerosis, neurodegenerative and psychiatric disorders (Perera and Turner, 2016; Rosso et al., 2016).

\section{In Body Weight}

Body weight of $\mathrm{NT5C} 1 \mathrm{~A}^{-/-}$and $\mathrm{NT} 5 \mathrm{C} 2^{-/-}$mice, fed a normal-chow diet, was similar to their WT littermates (Kviklyte et al., 2017). However, NT5C2 $2^{-1-}$ mice fed a high fat diet (HFD) increased their body weight significantly less as compared to WT mice (Johanns et al., 2019). The difference was not due to changes in food consumption or water intake. Although not significant, the authors reported a tendency toward increased AMPK activity in fat pads from $\mathrm{NT} 5 \mathrm{C} 2^{-/-}$compared with WT mice, both in basal and noradrenaline-stimulated conditions, while a significant increase in AMP concentration was only seen in fat pads from $N T 5 C 2^{-/-}$mice in response to noradrenaline treatment. Consistent with an activation of AMPK, a significant increase in ACC phosphorylation was associated to NT5C2 deletion and HFD (Johanns et al., 2019).

Genome-wide association studies performed on Japanese subjects revealed that the T-allele of rs11191548 in the NT5C2 gene was associated with reduced visceral fat area, subcutaneous fat area and total fat area in women (Hotta et al., 2012). Unfortunately, the authors did not measure the activity of NT5C2, therefore we do not know whether the reported single-nucleotide polymorphism affects the function of the enzyme and the level of AMP. Although not supported by the experimental data, it is conceivable to hypothesize an involvement of AMPK, which has been reported to integrate nutrient and hormonal signals to regulate food intake and body weight, both in the hypothalamus and peripheral tissues (Xue and Kahn, 2006). 


\section{CONCLUDING REMARKS}

The activity of NT5C2 is allosterically regulated by ATP that, at high physiological level, stabilises a very active enzyme conformation (Tozzi et al., 2013). In our opinion, high energy charge activates NT5C2 and AMP deaminase activities, leading to the hydrolysis of the excess of newly synthesized or salvaged nucleotides. At low energy charge, the low activity of both enzymes causes an accumulation of nucleoside monophosphates, particularly AMP, that can either activate AMPK, or be hydrolyzed by NT5C1, releasing adenosine, thus starting the purinergic signaling. In fact, extracellular adenosine, binding to widely distributed receptors (A1, A2A, A2B, and A3) acts not only on metabolic regulation through modulation of cyclicAMP intracellular concentration, but also on fine-tuning of synapses and on the coordination of neuronal networks (Agostinho et al., 2020). An increase of extracellular concentration of adenosine might reflect on many biological processes such as proliferation, regulation of blood flow, inflammation and immunosuppression (Vijayan et al., 2017; Jacobson et al., 2019). Conversely, in some cells or organs, the

\section{REFERENCES}

Agostinho, P., Madeira, D., Dias, L., Simões, A. P., Cunha, R. A., and Canas, P. M. (2020). Purinergic signaling orchestrating neuron-glia communication. Pharmacol. Res. 105253, 105253. doi:10.1016/j.phrs.2020.105253

Allegrini, S., Scaloni, A., Careddu, M. G., Cuccu, G., D’Ambrosio, C., Pesi, R., et al. (2004). Mechanistic studies on bovine cytosolic 5'-nucleotidase II, an enzyme belonging to the HAD superfamily. Eur. J. Biochem. 271, 4881-4891. doi:10. 1111/j.1432-1033.2004.04457.x

Barsotti, C., Pesi, R., Felice, F., and Ipata, P. L. (2003). The purine nucleoside cycle in cell-free extracts of rat brain: evidence for the occurrence of an inosine and a guanosine cycle with distinct metabolic roles. Cell. Mol. Life Sci. 60, 786-793. doi:10.1007/s00018-003-2371-x

Bester, A. C., Roniger, M., Oren, Y. S., Im, M. M., Sarni, D., Chaoat, M., et al. (2011). Nucleotide deficiency promotes genomic instability in early stages of cancer development. Cell 145, 435-446. doi:10.1016/j.cell.2011.03.044

Biever, A., Valjent, E., and Puighermanal, E. (2015). Ribosomal protein S6 phosphorylation in the nervous system: from regulation to function. Front. Mol. Neurosci. 8, 75. doi:10.3389/fnmol.2015.00075

Camici, M., Allegrini, S., and Tozzi, M. G. (2018). Interplay between adenylate metabolizing enzymes and AMP-activated protein kinase. FEBS J. 285, 3337-3352. doi:10.1111/febs.14508

Camici, M., Garcia-Gil, M., Pesi, R., Allegrini, S., and Tozzi, M. G. (2019). Purinemetabolising enzymes and apoptosis in cancer. Cancers 11, 1354. doi:10.3390/ cancers11091354

Coughlan, K. A., Valentine, R. J., Ruderman, N. B., and Saha, A. K. (2014). AMPK activation: a therapeutic target for type 2 diabetes? Diabetes Metab Syndr Obes 7, 241-253. doi:10.2147/DMSO.S43731

Cross-Disorder Group of the Psychiatric Genomics (2013). Identification of risk loci with shared effects on five major psychiatric disorders: a genome-wide analysis. Lancet 381, 1371-1379. doi:10.1016/S0140-6736(12)62129-1

Domise, M., Sauvé, F., Didier, S., Caillerez, R., Bégard, S., Carrier, S., et al. (2019). Neuronal AMP-activated protein kinase hyper-activation induces synaptic loss by an autophagy-mediated process. Cell Death Dis. 10, 221. doi:10.1038/s41419019-1464-x

Dreyer, H. C., Fujita, S., Cadenas, J. G., Chinkes, D. L., Volpi, E., and Rasmussen, B. B. (2006). Resistance exercise increases AMPK activity and reduces 4E-BP1 phosphorylation and protein synthesis in human skeletal muscle. J. Physiol. (Lond.) 576, 613-624. doi:10.1113/jphysiol.2006.113175 low NT5C2 activity obtained by silencing, was unable to produce significant AMP accumulation, casting some doubt on the mechanism linking low NT5C2 activity and its metabolic consequences. It will be very interesting to further investigate on these molecular mechanisms, since the knowledge of this matter will support the application of NT5C2 inhibitors not only in cancer but also in pathologies such as metabolic syndrome, obesity and diabetes.

\section{AUTHOR CONTRIBUTIONS}

MC, MG-G, and MT conceived the opinion and wrote the draft manuscript. SA and RP collected references and drew the figure. All authors discussed the content.

\section{FUNDING}

This work was supported by local grants of the University of Pisa (ex60\%2020).

Duarte, R. R. R., Bachtel, N. D., Côtel, M. C., Lee, S. H., Selvackadunco, S., Watson, I. A., et al. (2019). The psychiatric risk gene NT5C2 regulates adenosine monophosphate-activated protein kinase signaling and protein translation in human neural progenitor cells. Biol. Psychiatr. 86, 120-130. doi:10.1016/j. biopsych.2019.03.977

Duarte, R. R. R., Troakes, C., Nolan, M., Srivastava, D. P., Murray, R. M., and Bray, N. J. (2016). Genome-wide significant schizophrenia risk variation on chromosome 10q24 is associated with altered cis-regulation of BORCS7, AS3MT, and NT5C2 in the human brain. Am J Med Genet B Neuropsychiatr Genet 171, 806-814. doi:10.1002/ajmg.b.32445

Elsaid, M. F., Ibrahim, K., Chalhoub, N., Elsotouhy, A., El Mudehki, N., and Abdel Aleem, A. (2017). NT5C2 novel splicing variant expands the phenotypic spectrum of Spastic Paraplegia (SPG45): case report of a new member of thin corpus callosum SPG-Subgroup. BMC Med. Genet. 18, 33. doi:10.1186/ s12881-017-0395-6

Garcia, D., and Shaw, R. J. (2017). AMPK: mechanisms of cellular energy sensing and restoration of metabolic balance. Mol. Cell 66, 789-800. doi:10.1016/j. molcel.2017.05.032

Garcia-Gil, M., Pesi, R., Perna, S., Allegrini, S., Giannecchini, M., Camici, M., and Tozzi, M. G. (2003). 5'-aminoimidazole-4-carboxamide riboside induces apoptosis in human neuroblastoma cells. Neuroscience 117, 811-820. doi:10. 1016/s0306-4522(02)00836-9

Garcia-Gil, M., Camici, M., Allegrini, S., Pesi, R., Petrotto, E., and Tozzi, M. G. (2018). Emerging role of purine metabolizing enzymes in brain function and tumors. Int. J. Mol. Sci. 19, 3598. doi:10.3390/ijms19113598

Hardie, D. G., and Alessi, D. R. (2013). LKB1 and AMPK and the cancermetabolism link - ten years after. BMC Biol. 11, 36. doi:10.1186/1741-7007$11-36$

Hardie, D. G., Ross, F. A., and Hawley, S. A. (2012). AMPK: a nutrient and energy sensor that maintains energy homeostasis. Nat. Rev. Mol. Cell Biol. 13, 251-262. doi:10.1038/nrm3311

Hawley, S. A., Davison, M., Woods, A., Davies, S. P., Beri, R. K., Carling, D., et al. (1996). Characterization of the AMP-activated protein kinase kinase from rat liver and identification of threonine 172 as the major site at which it phosphorylates AMP-activated protein kinase. J. Biol. Chem. 271, 27879-27887. doi:10.1074/jbc.271.44.27879

Hawley, S. A., Pan, D. A., Mustard, K. J., Ross, L., Bain, J., Edelman, A. M., et al. (2005). Calmodulin-dependent protein kinase kinase-beta is an alternative upstream kinase for AMP-activated protein kinase. Cell Metabol. 2, 9-19. doi:10.1016/j.cmet.2005.05.009 
Hotta, K., Kitamoto, A., Kitamoto, T., Mizusawa, S., Teranishi, H., Matsuo, T., et al. (2012). Genetic variations in the CYP17A1 and NT5C2 genes are associated with a reduction in visceral and subcutaneous fat areas in Japanese women. J. Hum. Genet. 57, 46-51. doi:10.1038/jhg.2011.127

Hunsucker, S. A., Spychala, J., and Mitchell, B. S. (2001). Human cytosolic 5'nucleotidase I: characterization and role in nucleoside analog resistance. J. Biol. Chem. 276, 10498-10504. doi:10.1074/jbc.M011218200

Jacobson, K. A., Tosh, D. K., Jain, S., and Gao, Z. G. (2019). Historical and current adenosine receptor agonists in preclinical and clinical development. Front. Cell. Neurosci. 13, 124. doi:10.3389/fncel.2019.00124

Johanns, M., Kviklyte, S., Chuang, S. J., Corbeels, K., Jacobs, R., Herinckx, G., et al. (2019). Genetic deletion of soluble 5'-nucleotidase II reduces body weight gain and insulin resistance induced by a high-fat diet. Mol. Genet. Metabol. 126, 377-387. doi:10.1016/j.ymgme.2019.01.017

Kulkarni, S. S., Karlsson, H. K., Szekeres, F., Chibalin, A. V., Krook, A., and Zierath, J. R. (2011). Suppression of 5'-nucleotidase enzymes promotes AMP-activated protein kinase (AMPK) phosphorylation and metabolism in human and mouse skeletal muscle. J. Biol. Chem. 286, 34567-34574. doi:10.1074/jbc.M111.268292

Kviklyte, S., Vertommen, D., Yerna, X., Andersen, H., Xu, X., Gailly, P., et al. (2017). Effects of genetic deletion of soluble 5'-nucleotidases NT5C1A and NT5C2 on AMPK activation and nucleotide levels in contracting mouse skeletal muscles. Am. J. Physiol. Endocrinol. Metab. 313, E48-E62. doi:10.1152/ajpendo. 00304.2016

Perera, N. D., and Turner, B. J. (2016). AMPK signalling and defective energy metabolism in amyotrophic lateral sclerosis. Neurochem. Res. 41, 544-553. doi:10.1007/s11064-015-1665-3

Pesi, R., Micheli, V., Jacomelli, G., Peruzzi, L., Camici, M., Garcia-Gil, M., et al. (2000). Cytosolic 5'-nucleotidase hyperactivity in erythrocytes of Lesch-Nyhan syndrome patients. Neuroreport 11, 1827-1831. doi:10.1097/00001756200006260-00006

Pesi, R., Turriani, M., Allegrini, S., Scolozzi, C., Camici, M., Ipata, P. L., et al. (1994). The bifunctional cytosolic 5'-nucleotidase: regulation of the phosphotransferase and nucleotidase activities. Arch. Biochem. Biophys. 312, 75-80. doi:10.1006/ abbi.1994.1282

Pesi, R., Petrotto, E., Colombaioni, L., Allegrini, S., Garcia-Gil, M., Camici, M., et al. (2018). Cytosolic 5'-nucleotidase II silencing in a human lung carcinoma cell line opposes cancer phenotype with a concomitant increase in p53 phosphorylation. Int. J. Mol. Sci. 19, 2115. doi:10.3390/ijms19072115

Plaideau, C., Liu, J., Hartleib-Geschwindner, J., Bastin-Coyette, L., Bontemps, F., Oscarsson, J., et al. (2012). Overexpression of AMP-metabolizing enzymes controls adenine nucleotide levels and AMPK activation in HEK293T cells. Faseb. J. 26, 2685-2694. doi:10.1096/fj.11-198168

Rosso, P., Fioramonti, M., Fracassi, A., Marangoni, M., Taglietti, V., Siteni, S., et al. (2016). AMPK in the central nervous system: physiological roles and pathological implications. Res. Rep. Biol. 7, 1-13. doi:10.2147/Rrb.S90858
Sanders, M. J., Grondin, P. O., Hegarty, B. D., Snowden, M. A., and Carling, D. (2007). Investigating the mechanism for AMP activation of the AMPactivated protein kinase cascade. Biochem. J. 403, 139-148. doi:10.1042/ BJ20061520

Tkacz-Stachowska, K., Lechward, K., and Skladanowski, A. C. (2005). Isolation and characterization of pigeon breast muscle cytosolic 5'-nucleotidase-I (cN-I). Acta Biochim. Pol. 52, 789-796. doi:10.18388/abp.2005_3390

Tozzi, M. G., Pesi, R., and Allegrini, S. (2013). On the physiological role of cytosolic 5'-nucleotidase II (cN-II): pathological and therapeutical implications. Curr. Med. Chem. 20, 4285-4291. doi:10.2174/0929867311320340007

Vijayan, D., Young, A., Teng, M. W. L., and Smyth, M. J. (2017). Targeting immunosuppressive adenosine in cancer. Nat. Rev. Canc. 17 (12), 709-724. doi:10.1038/nrc.2017.86

Walldén, K., and Nordlund, P. (2011). Structural basis for the allosteric regulation and substrate recognition of human cytosolic 5'-nucleotidase II. J. Mol. Biol. 408, 684-696. doi:10.1016/j.jmb.2011.02.059

Williams, T., Courchet, J., Viollet, B., Brenman, J. E., and Polleux, F. (2011). AMPactivated protein kinase (AMPK) activity is not required for neuronal development but regulates axogenesis during metabolic stress. Proc. Natl. Acad. Sci. USA 108, 5849-5854. doi:10.1073/pnas.1013660108

Woods, A., Johnstone, S. R., Dickerson, K., Leiper, F. C., Fryer, L. G., Neumann, D., et al. (2003). LKB1 is the upstream kinase in the AMP-activated protein kinase cascade. Curr. Biol. 13, 2004-2008. doi:10.1016/j.cub.2003.10.031

Xiao, B., Heath, R., Saiu, P., Leiper, F. C., Leone, P., Jing, C., et al. (2007). Structural basis for AMP binding to mammalian AMP-activated protein kinase. Nature 449, 496-500. doi:10.1038/nature06161

Xue, B., and Kahn, B. B. (2006). AMPK integrates nutrient and hormonal signals to regulate food intake and energy balance through effects in the hypothalamus and peripheral tissues. J. Physiol. 574, 73-83. doi:10.1113/ jphysiol.2006.113217

Zhong, D., Guo, L., de Aguirre, I., Liu, X., Lamb, N., Sun, S. Y., et al. (2006). LKB1 mutation in large cell carcinoma of the lung. Lung Canc. 53, 285-294. doi:10. 1016/j.lungcan.2006.05.018

Conflict of Interest: The authors declare that the research was conducted in the absence of any commercial or financial relationships that could be construed as a potential conflict of interest.

Copyright (c) 2020 Camici, Garcia-Gil, Allegrini, Pesi and Tozzi. This is an openaccess article distributed under the terms of the Creative Commons Attribution License (CC BY). The use, distribution or reproduction in other forums is permitted, provided the original author(s) and the copyright owner(s) are credited and that the original publication in this journal is cited, in accordance with accepted academic practice. No use, distribution or reproduction is permitted which does not comply with these terms. 